\title{
New results for virial coefficients of hard spheres in $D$ dimensions
}

\author{
Nathan Clisby* \\ ARC Centre of Excellence for Mathematics and Statistics of Complex Systems \\ 139 Barry Street \\ The University of Melbourne, Victoria 3010 \\ Australia \\ Barry M. McCoy ${ }^{\dagger}$ \\ C. N. Yang Institute for Theoretical Physics \\ Stony Brook University \\ Stony Brook, NY 11794-3840
}

November 15, 2018

\begin{abstract}
We present new results for the virial coefficients $B_{k}$ with $k \leq 10$ for hard spheres in dimensions $D=2, \cdots, 8$.
\end{abstract}

YITP-SB-04-57

Keywords: hard spheres, virial expansion, Ree-Hoover diagrams

\section{Introduction}

The low density virial expansion of the pressure

$$
\frac{P}{k_{B} T}=\sum_{k=1}^{\infty} B_{k} \rho^{k} \quad \text { with } B_{1} \equiv 1
$$

for the hard sphere gas of particles of diameter $\sigma$ in $D$ dimensions defined by the two body potential

$$
U(\mathbf{r})=\left\{\begin{array}{cc}
+\infty & |\mathbf{r}|<\sigma \\
0 & |\mathbf{r}|>\sigma
\end{array}\right.
$$

is one of the oldest systems studied in statistical mechanics. The problem was first studied analytically by by van der Waals 1], Boltzmann [2], and van Laar [3] who computed the coefficients up through $B_{4}$. The computation of $B_{4}$ for $D=2$ was first done in 1964 by Rowlinson [4]

\footnotetext{
*e-mail:N.Clisby@ms.unimelb.edu.au

†e-mail:mccoy@insti.physics.sunysb.edu
} 
and Hemmer [5] and very recently these analytic computations for $B_{4}$ have been extended to $D=4,6,8,10,12$ by the present authors [6], and by Lyberg [7] for $D=5,7,9,11$.

All other computations for the hard sphere gas are by means of computer. This work was initiated in the 1950s for hard discs by Metropolis et al. [8] and for hard spheres by Rosenbluth and Rosenbluth 9]. Subsequently $B_{6}$ and $B_{7}$ were computed by Ree and Hoover [10, 11, 12] during the 1960s and $B_{8}$ was computed by Janse van Rensberg [13] in 1993. Computations for $D>3$ were initiated in 1964 by Ree and Hoover 14 who computed $B_{4}$ for $D=4, \cdots, 11$. The coefficients $B_{5}$ and $B_{6}$ for $D=4$ and 5 were computed by Bishop, Masters, and Clarke 15 in 1999, and Bishop, Masters, and Vlasov [16] have recently calculated $B_{7}$ in $D=4,5$ and $B_{8}$ in $D=4$.

In a series of papers [17, 18, 19] we have extended these numerical computations by computing virial coefficients up through $B_{10}$ in $D=2,3, \cdots, 8$. We use the method of Ree-Hoover diagrams as evaluated by Monte Carlo integration. The details are given in [19] Our results are given in Table 1 in the form of $B_{k} / B_{2}^{k-1}$ where

$$
B_{2}=\frac{\sigma^{D} \pi^{D / 2}}{2 \Gamma\left(1+\frac{D}{2}\right)}
$$

It is well known that for hard spheres in $D$ dimensions that for some sufficiently large $k$ which depends on $D$ that some Ree-Hoover diagrams for $B_{k}$ vanish identically for geometric reasons. We give (a lower bound on) the number of non-vanishing Ree-Hoover diagrams in Table 2

Table 2: Number of Mayer and Ree-Hoover integrals

\begin{tabular}{lccccccccc}
\hline & & & \multicolumn{7}{c}{ Order } \\
& 2 & 3 & 4 & 5 & 6 & 7 & 8 & 9 & 10 \\
\hline Mayer & 1 & 1 & 3 & 10 & 56 & 468 & 7123 & 194066 & 9743542 \\
RH & 1 & 1 & 2 & 5 & 23 & 171 & 2606 & 81564 & 4980756 \\
RH/Mayer & 1 & 1 & 0.667 & 0.500 & 0.410 & 0.365 & 0.366 & 0.420 & 0.511 \\
RH, $D=1$ & 1 & 1 & 1 & 1 & 1 & 1 & 1 & 1 & 1 \\
RH, $D=2$ & 1 & 1 & 2 & 4 & 15 & 73 & $\gtrsim 647$ & $\gtrsim 8417$ & $\gtrsim 110529$ \\
$\mathrm{RH}, D=3$ & 1 & 1 & 2 & 5 & 22 & 161 & $>2334$ & $>60902$ & \\
$\mathrm{RH}, D=4$ & 1 & 1 & 2 & 5 & 23 & 169 & $>2556$ & $>76318$ & \\
\hline
\end{tabular}

\section{Behavior of $B_{k}$ for large $k$}

It is apparent from Table 1 that negative virial coefficients occur. This was first observed for $B_{4}$ in 14. We observe that because $B_{4}$ changes sign between $D=7$ and $D=8, B_{6}$ changes sign between $D=5$ and 6 , and $B_{8}$ and $B_{10}$ change sign between $D=4$ and $D=5$. This suggests that for large $k$ the coefficient $B_{k}$ may become negative for dimensions smaller than 5 . In particular if for $D=2$ or $D=3$ there were a value of $k$ such that $B_{k}$ changed sign then approximate equations of state obtained from the first ten virial coefficients would be wholly inadequate to obtain the radius of convergence of the virial series.

The most important property of the virial coefficients $B_{k}$ is not their actual numerical values for $k$ less than some finite number but rather their asymptotic behavior as $k \rightarrow \infty$ because it is the asymptotic value which determines the radius of convergence. Of course no finite number of virial coefficients can give information on the $k \rightarrow \infty$ behavior unless there is some a priori reason to expect that the values of $k$ are already in the asymptotic $k \rightarrow \infty$ regime. 


\begin{tabular}{|c|c|c|c|c|c|c|c|c|}
\hline$D$ & $B_{3} / B_{2}^{2}$ & $B_{4} / B_{2}^{3}$ & $B_{5} / B_{2}^{4}$ & $B_{6} / B_{2}^{5}$ & $B_{7} / B_{2}^{6}$ & $B_{8} / B_{2}^{7}$ & $B_{9} / B_{2}^{8}$ & $B_{10} / B_{2}^{9}$ \\
\hline 2 & $0.782004 \cdots$ & $0.53223180 \ldots$ & $0.33355604(1)^{*}$ & $0.1988425(42)$ & $0.1148728(43)$ & $0.0649930(34)$ & $0.0362193(35)$ & $0.0199537(80)$ \\
\hline 3 & 0.625 & $0.2869495 \ldots$ & $0.110252(1)^{*}$ & $0.03888198(91)$ & $0.01302354(91)$ & $0.0041832(11)$ & $0.0013094(13)$ & $0.0004035(15)$ \\
\hline 4 & 0.506340 . & 0.15184606 . & $0.0357041(17)$ & $0.0077359(16)$ & $0.0014303(19)$ & $0.0002888(18)$ & $0.0000441(22)$ & $0.0000113(31)$ \\
\hline 5 & $0.414063 \ldots$ & $0.0759724807 \ldots$ & $0.0129551(13)$ & $0.0009815(14)$ & $0.0004162(19)$ & $-0.0001120(20)$ & $0.0000747(26)$ & $-0.0000492(48)$ \\
\hline 6 & $0.340941 \ldots$ & 0.03336314 . & $0.0075231(11)$ & $-0.0017385(13)$ & $0.0013066(18)$ & $-0.0008950(30)$ & $0.0006673(45)$ & $-0.000525(16)$ \\
\hline 7 & $0.282227 \ldots$ & $0.00986494662 \ldots$ & $0.0070724(10)$ & $-0.0035121(11)$ & $0.0025386(16)$ & $-0.0019937(28)$ & $0.0016869(41)$ & $-0.001514(14)$ \\
\hline 8 & 0.234614 . & $-0.00255768 \cdots$ & $0.00743092(93)$ & $-0.0045164(11)$ & $0.0034149(15)$ & $-0.0028624(26)$ & $0.0025969(38)$ & $-0.002511(13)$ \\
\hline
\end{tabular}

Table 1: Numerical values of virial coefficients. Values for $B_{7} D>5, B_{8} D>4, B_{9}$, and $B_{10}$ are new, and other values improve on published literature results for $B_{5}$ and higher except for the results for $B_{5}$ for $D=2,3$ which are due to Kratky [20]. 
We see in Table 2 the dramatic effect that the number of non-zero Ree-Hoover integrals in two dimensions is far less than that of the number of biconnected graphs with non-zero star content. At $k=10$ in $D=2$ we estimate that only 0.022 of the Ree-Hoover diagrams with non-zero coefficients have non-zero integrals.

The dramatic (at least in $D=2$ ) reduction as $k \rightarrow \infty$ in the number of non-vanishing ReeHoover diagrams gives a criteria for the size of $k$ needed for $B_{k}$ to be in the asymptotic region.

\section{Criteria 1}

The number of nonzero Ree Hoover diagrams has approached its large $k$ behavior.

For $k=10$ this criteria may only be fulfilled for $D=2$ and is surely not fulfilled at all for $D \geq 5$.

Our second criteria has been presented in our previous paper [17.

\section{Criteria 2}

The loose packed diagrams (defined to be those with the number of $\tilde{f}$ bonds near their maximum value) numerically dominate $B_{k}$ as $k \rightarrow \infty$.

The validity of this criteria has been studied in detail in [17. Here it was seen that for $D=3$ and $k \geq 12$ the criteria is well satisfied and that as $D$ increases the criteria is satisfied for smaller values of $k$. However, for $D=2$ the criteria was not satisfied even for $k$ as large as 17 .

We thus conclude that there is no dimension in which both of these criteria are simultaneously fulfilled though in $D=3$ and $D=4$ it is possible that they both could hold for some moderate values of $k$ such as $12-14$.

\section{Ratio Analysis}

Even though we have argued that $k=10$ may not be sufficiently large to see the true asymptotic behavior of $B_{k}$ it is still of interest to determine what results are obtained if well known methods are used to estimate the radius of convergence from the first ten virial coefficients.

One such way of estimating the radius of convergence is the analysis of the ratios of coefficients 21, 22] where we plot $B_{k} \rho_{c p} / B_{k-1}$ versus $1 / k$ (and we have normalized the virial coefficients to the density $\rho_{c p}$ of the closest packed lattice). The ratio extrapolated to $1 / k \rightarrow 0$ will give the radius of convergence of the series $\rho_{R}$ which may also expressed in terms of the packing fraction $\eta=B_{2} \rho / 2^{D-1}$. If the slope of the interpolated points approaches zero for large $k$ then the leading singularity is a pole on the positive real axis, if the slope is non-zero then the divergence is algebraic.

The plot of $B_{k} \rho_{c p} / B_{k-1}$ versus $\frac{1}{k}$ for $D=2$ is given in Fig. [1 Here we observe smoothly falling ratios which extrapolate to a radius of convergence greater that the closest packed density $\rho_{c p}$.

We plot the ratios for $D \geq 5$ in Figures 2 and 3 In this case the first few virial coefficients are positive, and then alternate in sign to the order calculated. We propose the scenario that there is a singularity on the positive real axis that dominates the series initially, but at higher order another singularity (or singularities) in the complex plane or negative real axis competes with the original singularity and hence the new singularity must be at a smaller radius. If the leading singularity is on the negative real axis then the ratio plot will smoothly converge to some negative value, otherwise the ratios will oscillate.

For $D=4$ in Fig. पit seems that despite the absence of negative virial coefficients and the poor accuracy of $B_{10}$, an oscillation is developing in the ratio plot in exactly the same way as for $D \geq 5$. Extrapolation of the series [19] via the methods of Dlog Padés and differential approximants as 
explained by Guttmann [22], suggests that negative coefficients for $D=4$ may occur for $k$ not much greater than 12 .

The case $D=3$ is plotted in Figures 1 and $[$ These ratios do not show the large oscillations of $D=4$ but close inspection reveals that the slopes are not increasing monotonically as they were for $D=2$. This may indicate that the plot for $D=3$ is displaying very small amplitude oscillations, which will eventually result in oscillations in the sign of the coefficients.

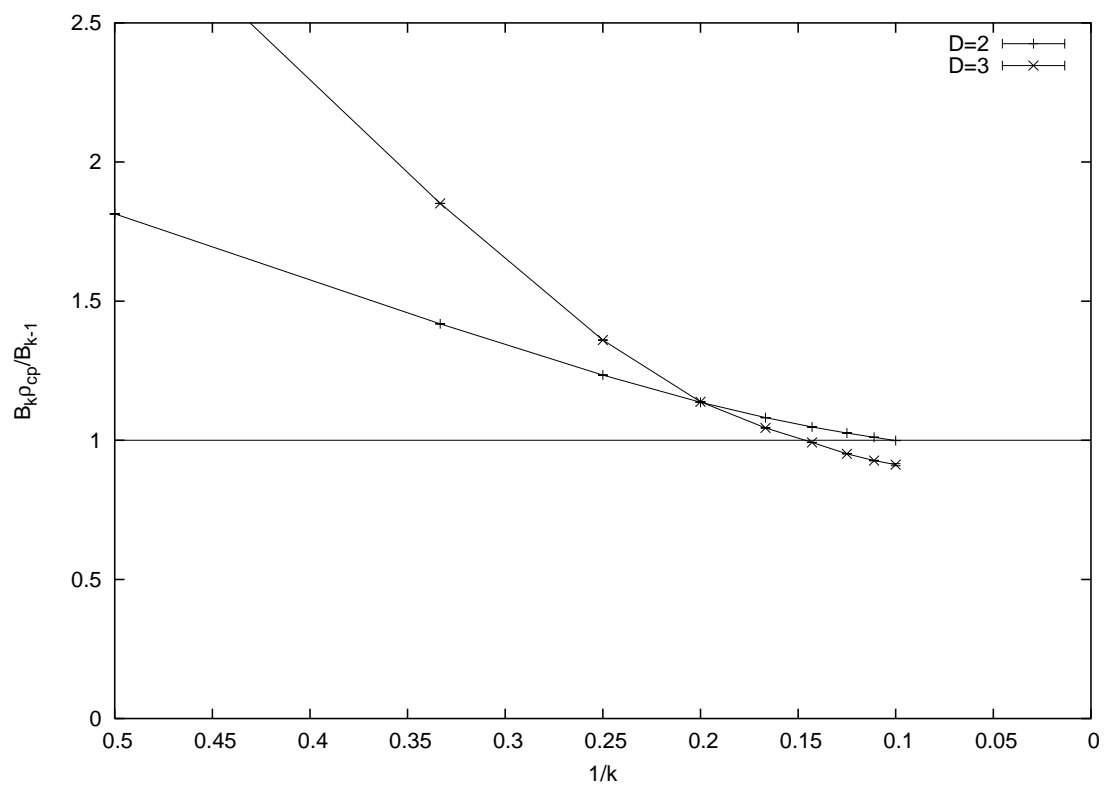

Figure 1: Ratio plot for virial coefficients in dimensions $D=2,3$

\section{Differential Approximants}

We have analyzed the virial coefficients of Table 1 by use of differential approximates using the fortran program NEWGRQD given in Guttmann [22]. Our results for the leading singularity on the real positive azis in dimensions $D=2,3,4$ are tabulated in Table 3 More detailed analysis will appear in [19. The notation $L, M ; N$ refers to an inhomogeneous first order differential approximant, which is the solution of

$$
z P_{M}(z) f^{\prime}(z)+Q_{L}(z) f(z)=R_{N}(z)
$$

where the subscript denotes the order of the polynomial, and $f(z)$ is the function that is to be approximated.

One can see from Tables 34 that there appears to be a singularity on the positive real axis close to the space filling density $\eta=1$ for dimensions $D=2,3,4$. The kind of singularity is not so clear, for $D=2$ there seems to be an algebraic singularity with exponent $\phi \simeq-1.75$, but for $D=3,4$ it is not possible to give a good estimate for the exponent. 


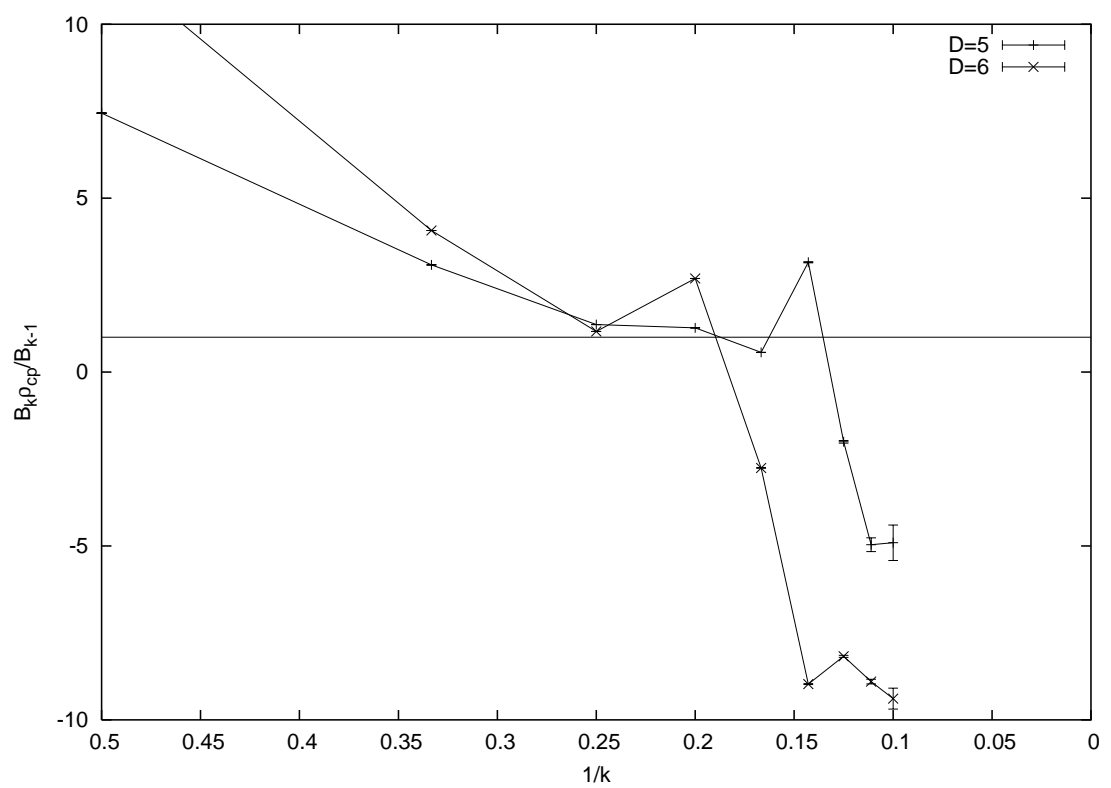

Figure 2: Ratio plot for virial coefficients in dimensions $D=5,6$

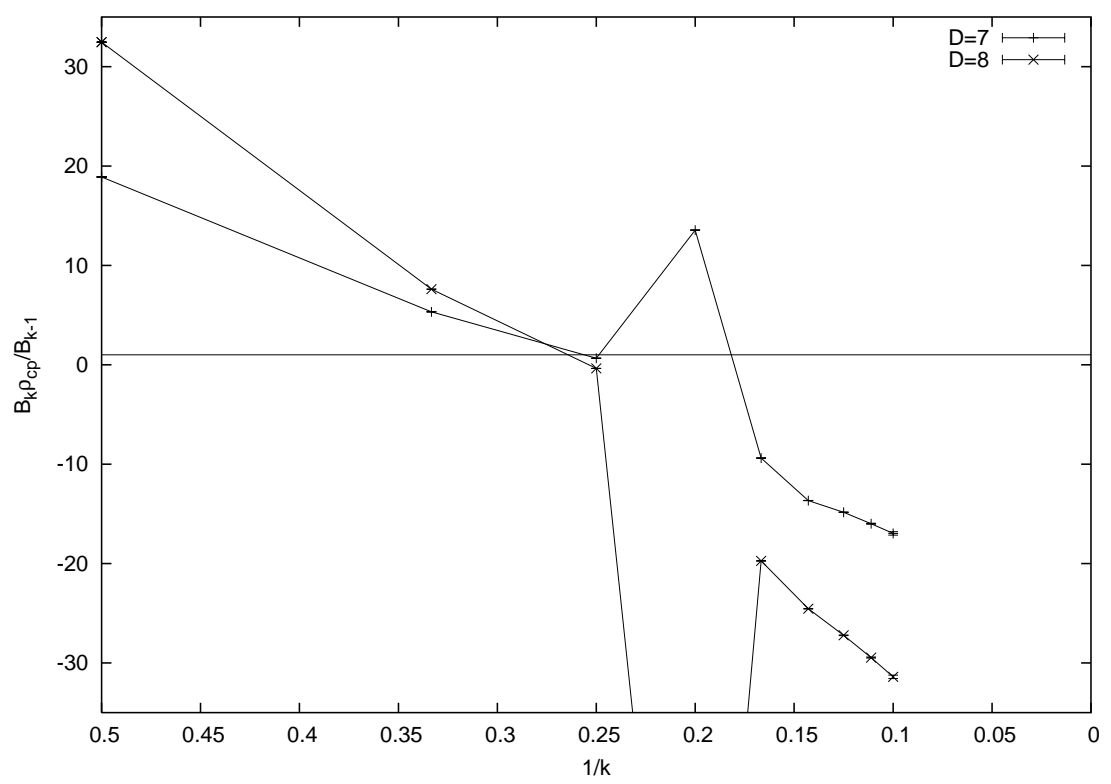

Figure 3: Ratio plot for virial coefficients in dimensions $D=7,8$

\section{Conclusion}

In Table 1above we have reported the first computations of the virial coefficients $B_{9}$ and $B_{10}$ for hard spheres in dimensions $D=2, \cdots, 8$, and shown that $B_{8}$ is negative for $D \geq 5$. The coefficient $B_{10}$ is negative for $D>4$ and at $D=4$ the ratios of sucessive coefficients oscillate in such a way 


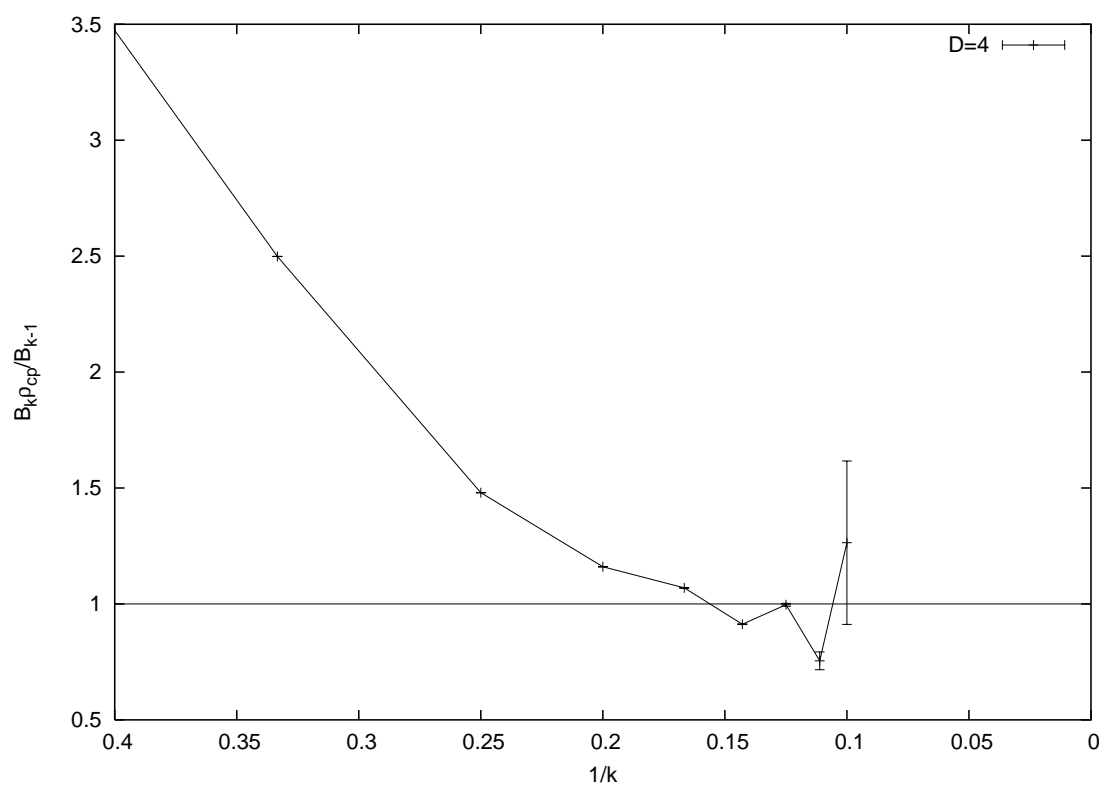

Figure 4: Ratio plot for virial coefficients in dimension $D=4$

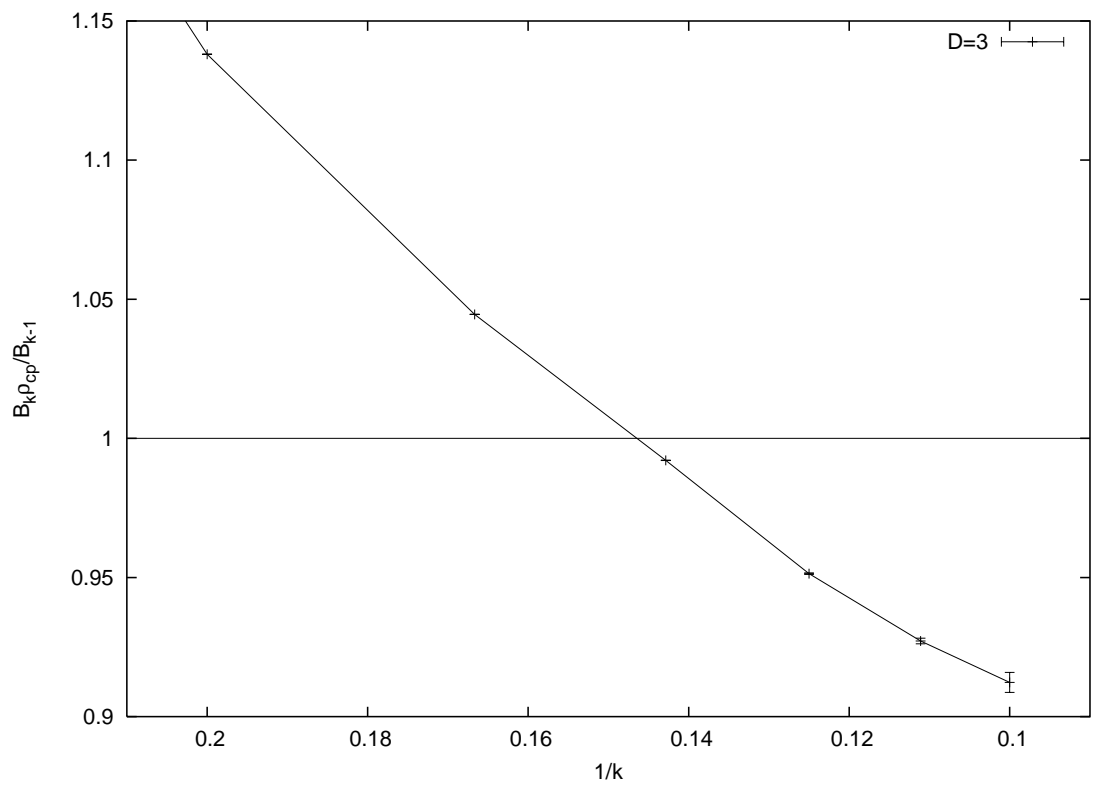

Figure 5: Ratio plot for virial coefficients in dimension $D=3$ over small domain to show non-monotonic behavior of the second derivative.

as to suggest that negative values may occur for $k=12-14$. For $D=2,3$ analysis of the first 10 virial coefficients leads to a radius of convergence greater than close packing. This is in agreement with conclusions reached in previous studies based on 8 or fewer virial coefficients. The meaning of this is controversial and the present authors argue that the true large $k$ behaviour is not seen 
Table 3: Singularities from differential approximants on the positive real axis for $D=2,3,4$. Blank entries are due to defective approximants.

\begin{tabular}{ccccccc}
\hline \multirow{2}{*}{$\begin{array}{c}\text { Differential } \\
\text { Approximant }\end{array}$} & $B_{2} \rho_{\text {sing }}$ & $\phi$ & \multicolumn{2}{c}{$D=3$} & \multicolumn{3}{c}{$D=4$} \\
A ${ }_{2} \rho_{\text {sing }}$ & $\phi$ & $B_{2} \rho_{\text {sing }}$ & $\phi$ \\
\hline 3,$3 ; 0$ & 1.987 & -1.790 & 4.068 & -2.818 & 7.995 & -3.520 \\
3,$4 ; 0$ & 1.984 & -1.774 & 3.830 & -2.329 & 6.843 & -2.478 \\
4,$3 ; 0$ & 1.984 & -1.774 & 3.714 & -2.043 & 5.551 & -1.207 \\
4,$4 ; 0$ & 1.987 & -1.788 & 3.732 & -2.090 & 7.249 & -2.871 \\
4,$5 ; 0$ & 1.988 & -1.795 & 3.675 & -1.899 & 6.985 & -2.583 \\
5,$4 ; 0$ & 1.988 & -1.795 & 3.720 & -2.056 & 6.721 & -2.229 \\
2,$2 ; 1$ & 1.946 & -1.575 & 3.659 & -2.014 & 6.888 & -2.593 \\
2,$3 ; 1$ & 1.970 & -1.695 & 3.787 & -2.246 & 5.412 & -2.922 \\
3,$2 ; 1$ & 1.966 & -1.677 & 4.038 & -2.953 & 8.332 & -4.368 \\
3,$3 ; 1$ & 2.021 & -2.076 & 3.811 & -2.298 & 7.296 & -2.920 \\
3,$4 ; 1$ & 1.981 & -1.756 & 3.786 & -2.241 & 6.764 & -2.389 \\
4,$3 ; 1$ & 1.978 & -1.740 & 3.708 & -2.024 & 4.663 & -3.708 \\
2,$1 ; 2$ & 1.945 & -1.572 & 3.676 & -2.054 & $\ldots$ & $\ldots$ \\
2,$2 ; 2$ & 1.967 & -1.682 & 3.641 & -1.987 & 7.715 & -3.531 \\
2,$3 ; 2$ & 2.008 & -1.900 & 3.799 & -2.269 & 7.277 & -2.916 \\
3,$2 ; 2$ & $\ldots$ & $\ldots$ & 3.874 & -2.468 & 7.250 & -2.899 \\
3,$3 ; 2$ & 1.971 & -1.679 & 3.773 & -2.210 & 7.308 & -2.920 \\
2,$1 ; 3$ & 1.959 & -1.628 & 3.599 & -1.889 & 6.802 & -2.680 \\
2,$2 ; 3$ & 1.984 & -1.784 & 3.747 & -1.956 & 7.508 & -3.404 \\
2,$3 ; 3$ & 1.982 & -1.778 & 3.777 & -2.110 & 7.003 & -2.539 \\
3,$2 ; 3$ & 1.981 & -1.770 & 3.779 & -2.034 & $\ldots$ & $\ldots$ \\
\hline
\end{tabular}

Table 4: Approximate position of singularities with exponents

\begin{tabular}{llll}
\hline$D$ & $B_{2} \rho_{\text {sing }}$ & $\eta_{\text {sing }}$ & \multicolumn{1}{c}{$\phi$} \\
\hline 1 & 1.00 & 1.00 & -1.00 \\
2 & 1.98 & 0.99 & -1.75 \\
3 & 3.75 & 0.94 & -2.1 \\
4 & 7.00 & 0.88 & -3 \\
\hline
\end{tabular}

in the first 10 coefficients. More complete analysis and discussion of this is given in [19].

Acknowledgments: This work was supported in part by the National Science Foundation under DMR-0302758.

\section{References}

[1] J. D. van der Waals. Proc. Kon. Acad. V. Wetensch, Amsterdam, 1:138, 1899. 
[2] L. Boltzmann. Proc. Sect. Sci. K. Akad. Wet. (Amsterdam), 1899.

[3] J. J. van Laar. Proc. Kon. Acad. V. Wetensch, Amsterdam, 1:273, 1899.

[4] J. S. Rowlinson. The virial expansion in two dimensions. Mol. Phys., 7:593-594, 1964.

[5] P. C. Hemmer. Virial Coefficients for the Hard-Core Gas in Two Dimensions. J. Chem. Phys., 42:1116-1118, 1964.

[6] N. Clisby and B. M. McCoy. Analytical calculation of $B_{4}$ for hard spheres in even dimensions. J. Stat. Phys., 114:1343-1361, 2004.

[7] I. Lyberg. The fourth virial coefficient of a fluid of hard spheres in odd dimensions. cond-mat/0410080 2004.

[8] N. Metropolis, A. W. Rosenbluth, M. N. Rosenbluth, and A. H. Teller. Equation of State Calculations by Fast Computing Machines. J. Chem. Phys., 21:1087-1092, 1953.

[9] M. N. Rosenbluth and A. W. Rosenbluth. Further Results on Monte Carlo Equations of State. J. Chem. Phys., 22:881-884, 1954.

[10] F. H. Ree and W. G. Hoover. Fifth and sixth virial coefficients for hard spheres and hard discs. J. Chem. Phys., 40:939-950, 1964.

[11] F. H. Ree and W. G. Hoover. Reformulation of the Virial Series for Classical Fluids. J. Chem. Phys., 41:1635-1645, 1964.

[12] F. H. Ree and W. G. Hoover. Seventh virial coefficients for hard spheres and hard discs. J. Chem. Phys., 46:4181-4196, 1967.

[13] E. J. Janse van Rensburg. Virial coefficients for hard discs and hard spheres. J. Phys. A, 26:4805-4818, 1993.

[14] F. H. Ree and W. G. Hoover. On the signs of the hard sphere virial coefficients. J. Chem. Phys., 40:2048-2049, 1964.

[15] M. Bishop, A. Masters, and J. H. R. Clarke. Equation of state of hard and Weeks-ChandlerAnderson hyperspheres in four and five dimensions. J. Chem. Phys., 110:11449-11453, 1999.

[16] M. Bishop, A. Masters, and A. Y. Vlasov. Higher virial coefficients of four and five dimensional hard hyperspheres. J. Chem. Phys., 121:6884-6886, 2004.

[17] N. Clisby and B. M. McCoy. Negative virial coefficients and the dominance of loose packed diagrams for D-dimensional hard spheres. J. Stat. Phys., 114:1361-1392, 2004.

[18] N. Clisby. Negative Virial Coefficients for Hard Spheres. PhD thesis, Stony Brook University, Stony Brook, New York, 2004.

[19] N. Clisby and B. M. McCoy. Ninth and Tenth Order Virial Coefficients for Hard Spheres in $D$ Dimensions, in preparation.

[20] K. W. Kratky. A New Graph Expansion of Virial Coefficients. J. Stat. Phys., 27:533-551, 1982. 
[21] D. S. Gaunt and G. S. Joyce. Virial expansions for hard-core fluids. J. Phys. A, 13:L211-L216, 1980.

[22] A. J. Guttmann. Asymptotic Analysis of Power-Series Expansions. In C. Domb and J. Lebowitz, editors, Phase Transitions and Critical Phenomena, volume 13, chapter 1, pages 1-234. Academic Press, 1989. 